Supporting Information

\title{
Characteristics of the isolated products
}

1-Benzyl-4-phenyl-1H-1,2,3-triazole (3a): ${ }^{[1]}$<smiles>c1ccc(Cn2cc(-c3ccccc3)nn2)cc1</smiles>

white solid (crystals), melting point: $126-128{ }^{\circ} \mathrm{C}$; IR: $\tilde{v}=3029-3143$ (=C-H-valence), 1450 ( $\mathrm{CH}_{2}, \mathrm{CH}_{3}$-deform.), 728, 695 (=C-H-deform.) $\mathrm{cm}^{-1}$; MS (EI): $\mathrm{m} / \mathrm{z}$ (\%): 235 (21) [M $\left.\mathrm{M}^{+}\right], 206$ (82), 116 (100), 104 (19), 91 (82), 89 (32), 65 (19); ${ }^{1} \mathrm{H}$ NMR (400 MHz, CDCl $): \delta=7.80(2 \mathrm{H}, \mathrm{d}, \mathrm{J}$ $=7,6 \mathrm{~Hz}), 7.71(1 \mathrm{H}, \mathrm{s}), 7.40-7.24(8 \mathrm{H}, \mathrm{m}), 5.56(2 \mathrm{H}, \mathrm{s}) \mathrm{ppm} ;{ }^{13} \mathrm{C} \mathrm{NMR}\left(100 \mathrm{MHz}, \mathrm{CDCl}_{3}\right): \delta=$ $134.48,130.13,129.13,128.80,128.28,128.07,125.73,119.86,54.35 \mathrm{ppm}$.

1-(Benzyl)-4-(4-methylphenyl)-1H-1,2,3-triazole (3b): $:^{[1]}$<smiles>Cc1ccc(-c2cn(Cc3ccccc3)nn2)cc1</smiles>

white solid (crystals), melting point: $151-153^{\circ} \mathrm{C}$; IR: $\approx=3146,3019$ (=C-H-valence), 29602855 ( $\mathrm{CH}_{2}, \mathrm{CH}_{3}$-valence), 1456 ( $\mathrm{CH}_{2}, \mathrm{CH}_{3}$-deform.), 828, 793 (=C-H-deform.) $\mathrm{cm}^{-1}$; MS (EI): m/z (\%): 249 (23) [M+], 221 (15), 220 (81), 206 (20), 179 (18), 130 (100), 103 (20), 91 (71), 77 (17), 65 (15); ${ }^{1} \mathrm{H}$ NMR $\left(200 \mathrm{MHz}, \mathrm{CDCl}_{3}\right): \delta=7.68(2 \mathrm{H}, \mathrm{m}), 7.37-7.16(8 \mathrm{H}, \mathrm{m}), 5.53(2 \mathrm{H}$, 
s) $2.33(3 \mathrm{H}, \mathrm{s}) \mathrm{ppm} ;{ }^{13} \mathrm{C} \mathrm{NMR}\left(50 \mathrm{MHz}, \mathrm{CDCl}_{3}\right)$ : $\delta=138.05,134.59,129.45,129.08,128.73$, 128.04, 125.57, 54.27, $21.23 \mathrm{ppm}$. 
1-(Benzyl)-4-(4-methoxyphenyl)-1H-1,2,3-triazole (3c): $:^{[1 \mathrm{a}]}$<smiles>COc1ccc(-c2cn(Cc3ccccc3)nn2)cc1</smiles>

white solid (crystals), melting point: $138-141^{\circ} \mathrm{C}$; IR: $\tilde{v}=3138,3038$ (=C-H-valence), 2928$2838\left(\mathrm{CH}_{2}, \mathrm{CH}_{3}\right.$-valence), 1455 ( $\mathrm{CH}_{2}, \mathrm{CH}_{3}$-deform. $), 834,795,718,700$ (=C-H-deform.) $\mathrm{cm}^{-1}$; MS (EI): m/z (\%): 265 (32) [M+], 237 (20), 236 (100), 222 (16), 209 (20), 206 (19), 146 (79), 119 (25), 91 (57), 65 (18); ${ }^{1} \mathrm{H}$ NMR (600 MHz, $\left.\mathrm{CDCl}_{3}\right): \delta=7.71(2 \mathrm{H}, \mathrm{d}, \mathrm{J}=8.451 \mathrm{~Hz}), 7.62$ $(1 \mathrm{H}, \mathrm{s}), 7.38-7.28(5 \mathrm{H}, \mathrm{m}), 6.91(2 \mathrm{H}, \mathrm{d}, \mathrm{J}=8.019 \mathrm{~Hz}), 5.53(2 \mathrm{H}, \mathrm{s}), 3.79(3 \mathrm{H}, \mathrm{s}) \mathrm{ppm} ;{ }^{13} \mathrm{C}$ NMR $\left(150 \mathrm{MHz}, \mathrm{CDCl}_{3}\right): \delta=159.64,134.63,133.53,129.09,128.72,128.03,127.02$, $123.01,114.20,113.89,55.25,54.26$ ppm.

1-Benzyl-4-octyl-1H-1,2,3-triazole (3d): $:^{[1]}$

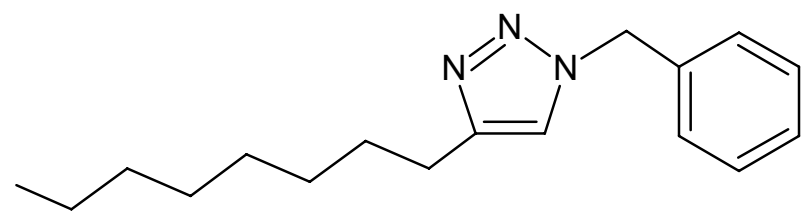

slightly green solid (crystals), melting point: $69-70{ }^{\circ} \mathrm{C}$; IR: $\vec{v}=3065-3143$ (=C-H-valence), 2955-2850 ( $\mathrm{CH}_{2}, \mathrm{CH}_{3}$-valence), 1450 ( $\mathrm{CH}_{2}, \mathrm{CH}_{3}$-deform.), 730, 694 (=C-H-deform.) cm ${ }^{-1}$; MS (EI): $m / z(\%): 271$ (6) [M+], 173 (11), 92 (9), 91 (100), 172 (7), 65 (7); ${ }^{1} \mathrm{H}$ NMR (200 MHz, $\left.\mathrm{CDCl}_{3}\right): \delta=7.34-7.19(6 \mathrm{H}, \mathrm{m}), 5.47(2 \mathrm{H}, \mathrm{s}), 2.65(1 \mathrm{H}, \mathrm{s}), 1.61(1 \mathrm{H}, \mathrm{s}), 1.21(10 \mathrm{H}, \mathrm{s}), 0,83$ $(5 \mathrm{H}, \mathrm{t}) \mathrm{ppm} ;{ }^{13} \mathrm{C} \mathrm{NMR}\left(50 \mathrm{MHz}, \mathrm{CDCl}_{3}\right): \delta=134.35,128.70,128.34,127.67,54.08,31.44$, $28.91,28.83,25.23,22.27,13.72 \mathrm{ppm}$.

1-Benzyl-4-decyl-1H-1,2,3-triazole (3e): ${ }^{[2]}$

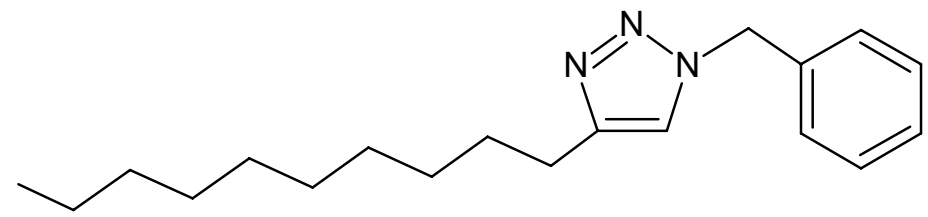

bright beige solid (powder), melting point: $77-78^{\circ} \mathrm{C}$; IR: $\tilde{v}=3065-3144$ (=C-H-valence), 2955-2849 ( $\mathrm{CH}_{2}, \mathrm{CH}_{3}$-valence), 1470, $1454\left(\mathrm{CH}_{2}, \mathrm{CH}_{3}\right.$-deform. $), 720,703$ (=C-H-deform. $) \mathrm{cm}^{-}$ 1; MS (EI): m/z (\%): 299 (8) [M+], 180 (8), 173 (12), 172 (9), 92 (8), 91 (100); ${ }^{1} \mathrm{H}$ NMR $\left(400 \mathrm{MHz}, \mathrm{CDCl}_{3}\right): \delta=7.35-7.22(6 \mathrm{H}, \mathrm{m}), 5.50(2 \mathrm{H}, \mathrm{s}), 2.67(1 \mathrm{H}, \mathrm{s}), 1.65(1 \mathrm{H}, \mathrm{s}), 1.23(20 \mathrm{H}$, s), 0,85 (5H, t) ppm; $\left.{ }^{13} \mathrm{C} \mathrm{NMR} \mathrm{(100} \mathrm{MHz,} \mathrm{CDCl}_{3}\right): \delta=134.59,129.03,128.72,128.06,84.78$, $67.97,31.84,29.52,29.47,29.27,29.20,28.45,22.62,18.35,14.06$ ppm. 
2-(1-benzyl-1H-1,2,3-triazole-4-yl)propan-2-ol (3f): $:^{[3]}$<smiles>CC(C)(O)c1cn(Cc2ccccc2)nn1</smiles>

bright brown solid (powder), melting point: $74-76{ }^{\circ} \mathrm{C}$; IR: $\tilde{w}=3298$ (OH-valence), 3144 (=CH-valence), 2977-2850 ( $\mathrm{CH}_{2}, \mathrm{CH}_{3}$-valence), $1452\left(\mathrm{CH}_{2}, \mathrm{CH}_{3}\right.$-deform.), 1172 (C-O-valence), 729, 696 (=C-H-deform.) cm ${ }^{-1}$; MS (EI): m/z (\%): 217 (<1) [M+1], 202 (30), 170 (12), 92 (8), 91 (100), 80 (8), 65 (13); ${ }^{1} \mathrm{H}$ NMR (200 MHz, $\left.\mathrm{CDCl}_{3}\right): \delta=7.34-7.20(6 \mathrm{H}, \mathrm{m}), 5.43(2 \mathrm{H}, \mathrm{s}), 1.66$ $(6 \mathrm{H}, \mathrm{s}) \mathrm{ppm} ;{ }^{13} \mathrm{C} \mathrm{NMR}\left(50 \mathrm{MHz}, \mathrm{CDCl}_{3}\right): \delta=134.08,128.74,128.72,128.47,127.90,30.93$, $22.20,13.69 \mathrm{ppm}$.

2-(1-benzyl-1H-1,2,3-triazole-4-yl)but-3-en-2-ol (3g):<smiles>C=CC(C)(O)c1cn(Cc2ccccc2)nn1</smiles>

red-brown oil; IR: $\tilde{v}=3373$ (OH-valence), 2980-2855 $\left(\mathrm{CH}_{2}, \mathrm{CH}_{3}\right.$-valence), $1456\left(\mathrm{CH}_{2}, \mathrm{CH}_{3}\right.$ deform.), 1139 (C-O-valence), 718, 693 (=C-H-deform.) cm ${ }^{-1}$; MS (EI): m/z (\%): 229 (<1) $\left[\mathrm{M}^{+}\right], 212$ (11), 186 (36), 92 (9), 91 (100), 65 (14); ${ }^{1} \mathrm{H}$ NMR (400 MHz, $\mathrm{CDCl}_{3}$ ): $\delta=7.37-7.22$ $(6 \mathrm{H}, \mathrm{m}), 6.22(1 \mathrm{H}, \mathrm{s}), 5.45(2 \mathrm{H}, \mathrm{s}), 5.30(1 \mathrm{H}, \mathrm{s}), 5.15(1 \mathrm{H}, \mathrm{s}), 1.69(3 \mathrm{H}, \mathrm{s}) \mathrm{ppm},{ }^{13} \mathrm{C} \mathrm{NMR}$ $\left(100 \mathrm{MHz}, \mathrm{CDCl}_{3}\right): \delta=143.26,134.42,129.10,128.76,128.15,127.98,122.10,112.66$, $54.14,40.24,31.59,28.62,22.59,14.01 \mathrm{ppm}$.

2-(1-benzyl-1H-1,2,3-triazole-4-yl)-6-methylhept-5-en-2-ol (3h):

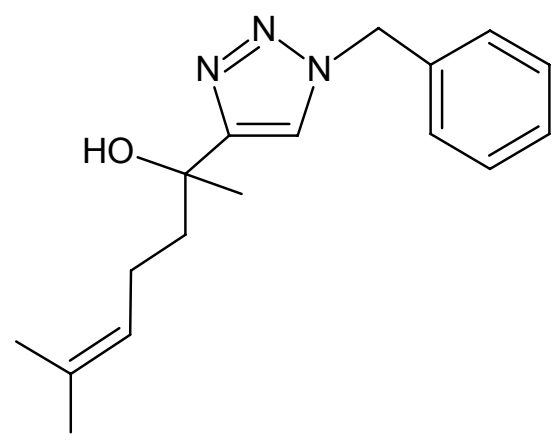

green oil; IR: $\tilde{v}=3382$ (OH-valence), $2970-2856\left(\mathrm{CH}_{2}, \mathrm{CH}_{3}\right.$-valence), $1456\left(\mathrm{CH}_{2}, \mathrm{CH}_{3}-\right.$ deform.), 1176, 1124 (C-O-valence), 720, 695 (=C-H-deform.) cm ${ }^{-1}$; MS (EI): m/z (\%): 285 (1) [M+'], 267 (20), 203 (38), 112 (16), 92 (16), 91 (100), 65 (12); ${ }^{1} \mathrm{H} \mathrm{NMR} \mathrm{(200} \mathrm{MHz,} \mathrm{CDCl}_{3}$ ): $\delta=$ 7.35-7.20 (6H, m), $5.45(2 \mathrm{H}, \mathrm{s}), 5.06(1 \mathrm{H}, \mathrm{s}), 1.94(2 \mathrm{H}, \mathrm{m}), 1.60(6 \mathrm{H}, \mathrm{m}), 1.46(4 \mathrm{H}, \mathrm{m}), 1.23$ 
$(1 \mathrm{H}, \mathrm{s}) \mathrm{ppm} ;{ }^{13} \mathrm{C}$ NMR $\left(50 \mathrm{MHz}, \mathrm{CDCl}_{3}\right): \delta=134.45,132.10,129.05,128.77,128.12,123.85$, $123.61,87.56,71.37,68.18,43.09,29.74,25.60,23.51,22.74,17.56$ ppm.

Methyl 1-benzyl-1H-1,2,3-triazole-4-carboxylate (3i): ${ }^{[4]}$<smiles>COC(=O)c1cn(Cc2ccccc2)nn1</smiles>

Dark brown solid (powder), melting point: $107^{\circ} \mathrm{C}$; IR: $\tilde{v}=3112,3070,3003$ (=C-H-valence), 2952-2855 ( $\mathrm{CH}_{2}, \mathrm{CH}_{3}$-valence), 1721 (-C=O-valence), 1456, $1433\left(\mathrm{CH}_{2}, \mathrm{CH}_{3}\right.$-deform.), 1226, 1047, 1019 (-C-O-C-valence), 780, 712, 692 (=C-H-deform.) cm ${ }^{-1}$; MS (EI): m/z (\%): 217 (1) $\left[\mathrm{M}^{+}\right], 188$ (14), 174 (27), 131 (10), 130 (26), 91 (100), 65 (15); ${ }^{1} \mathrm{H}$ NMR (200 MHz, $\mathrm{CDCl}_{3}$ ): $\delta$ $=7.98(1 \mathrm{H}, \mathrm{s}), 7.36-7.19(5 \mathrm{H}, \mathrm{m}), 5.51(2 \mathrm{H}, \mathrm{s}), 3.86(3 \mathrm{H}, \mathrm{s}) \mathrm{ppm} ;{ }^{13} \mathrm{C} \mathrm{NMR}\left(50 \mathrm{MHz}, \mathrm{CDCl}_{3}\right)$ : $\delta=161.01,133.56,129.24,129.09,128.30,128.22,54.47,52.13,22.51$ ppm.

Dimethyl 1-benzyl-1H-1,2,3-triazole-4,5-dicarboxylate $(\mathbf{3 j}):^{[5]}$<smiles>COC(=O)c1nnn(Cc2ccccc2)c1C(=O)OC</smiles>

Dark brown oil; IR: $\tilde{v}=$ 2952-2857 ( $\mathrm{CH}_{2}, \mathrm{CH}_{3}$-valence), 1727 (-C=O-valence), 1456, 1435 $\left(\mathrm{CH}_{2}, \mathrm{CH}_{3}\right.$-deform.), 1213, 1059, (-C-O-C-valence), 719, 695 (=C-H-deform.) $\mathrm{cm}^{-1}$; MS (EI): m/z (\%): $275(-)\left[\mathrm{M}^{+}\right], 214$ (11), 156 (12), 130 (15), 129 (12), 91 (100), 65 (13); ${ }^{1} \mathrm{H}$ NMR $\left(400 \mathrm{MHz}, \mathrm{CDCl}_{3}\right): \delta=7.31-7.29(3 \mathrm{H}, \mathrm{m}), 7.24-7.21(2 \mathrm{H}, \mathrm{m}), 5.58(2 \mathrm{H}, \mathrm{s}), 3.92(3 \mathrm{H}, \mathrm{s}), 3.90$ $(3 \mathrm{H}, \mathrm{s}) \mathrm{ppm} ;{ }^{13} \mathrm{C} \mathrm{NMR}\left(100 \mathrm{MHz}, \mathrm{CDCl}_{3}\right): \delta=160.38,158.79,133.85,130.60,129.28$, 128.91, 128.80, 128.25, 127.96, 54.75, 53.90, 53.27, 52.66, 52.15, 31.60, 29.19, 22.53 ppm.

Methyl 1-benzyl-1H-1,2,3-triazole-4-carboxylate (3i; from reaction of $\mathbf{2 k}):^{[4]}$<smiles>COC(=O)c1cn(Cc2ccccc2)nn1</smiles>

brown solid (powder), melting point: $87-88^{\circ} \mathrm{C}$; IR: $\tilde{v}=3117-3009$ (=C-H-valence), 2959-2857 ( $\mathrm{CH}_{2}, \mathrm{CH}_{3}$-valence), 1719 (-C=O-valence), 1457, 1435 ( $\mathrm{CH}_{2}, \mathrm{CH}_{3}$-deform.), 1229, 1045, 1016

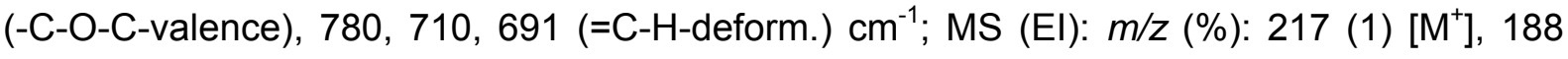


(16), 174 (30), 131 (11), 130 (27), 91 (100), 65 (15); ${ }^{1} \mathrm{H}$ NMR (200 MHz, $\left.\mathrm{CDCl}_{3}\right): \delta=7.99$ $(1 \mathrm{H}, \mathrm{s}), 7.39-7.23(5 \mathrm{H}, \mathrm{m}), 5.55(2 \mathrm{H}, \mathrm{s}), 3.89(3 \mathrm{H}, \mathrm{s}) \mathrm{ppm} ;{ }^{13} \mathrm{C} \mathrm{NMR}\left(50 \mathrm{MHz}, \mathrm{CDCl}_{3}\right): \delta=$ $161.03,133.58,129.27,129.12,128.24,54.48,52.15,14.04$ ppm.

1-Decyl-4-phenyl-1H-1,2,3-triazole $(3 \mathrm{~m}):^{[1 \mathrm{~b}]}$

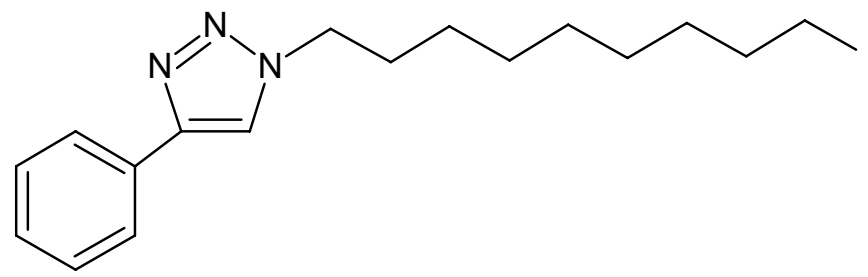

white solid (crystals), melting point: $63-66^{\circ} \mathrm{C}$; IR: $\tilde{V}=3121,3093,3065$ (=C-H-valence), 2956-2848 ( $\mathrm{CH}_{2}, \mathrm{CH}_{3}$-valence), $1463\left(\mathrm{CH}_{2}, \mathrm{CH}_{3}\right.$-deform.), 761, 695 (=C-H-deform.) cm ${ }^{-1}$; MS (EI): m/z (\%): 285 (28) [M+], 256 (32), 172 (29), 145 (35), 144 (22), 130 (17), 117 (100), 116 (26), 104 (33), 102 (16); ${ }^{1} \mathrm{H}$ NMR (400 MHz, $\left.\mathrm{CDCl}_{3}\right): \delta=7.87(2 \mathrm{H}, \mathrm{d}, \mathrm{J}=7,5 \mathrm{~Hz}), 7.85(1 \mathrm{H}$, s), 7.46-7.34 (3H, m), $4.40(2 \mathrm{H}, \mathrm{t}), 1.96(2 \mathrm{H}, \mathrm{m}), 1.31-1.21(14 \mathrm{H}, \mathrm{m}), 0.89(3 \mathrm{H}, \mathrm{m}) \mathrm{ppm} ;{ }^{13} \mathrm{C}$ NMR $\left(100 \mathrm{MHz}, \mathrm{CDCl}_{3}\right): \delta=130.65,128.85,128.13,125.70,50.53,31.86,30.36,29.47$, 29.39, 29.26, 29.02, 26.73, 26.51, 22.67, 14.12 ppm.

\section{References}

[1] a) K. Kamata, Y. Nakagawa, K. Yamaguchi, N. Mizuno, J. Am. Chem. Soc. 2008, 130, 15304-15310; b) T. Miao, L. Wang, Synthesis 2008, 363-368.

[2] J. Doiron, A. H. Soultan, R. Richard, M. M. Touré, N. Picot, R. Richard, M. Čuperlović-Culf, G. A. Robichaud, M. Touaibia, Eur. J. Med. Chem. 2011, 46, 40104024.

[3] P. Veerakumar, M. Velayudham, K.-L. Lu, S. Rajagopal, Catal. Sci. Technol. 2011, 1, 1512-1525.

[4] K. Asano, S. Matsubara, Org. Lett. 2010, 12, 4988-4991.

[5] S. E. Metobo, H. Jin, M. Tsiang, C. U. Kim, Bioorg. Med. Chem. Lett. 2006, 16, 39853988. 
1-Benzyl-4-phenyl-1H-1,2,3-triazole (3a):
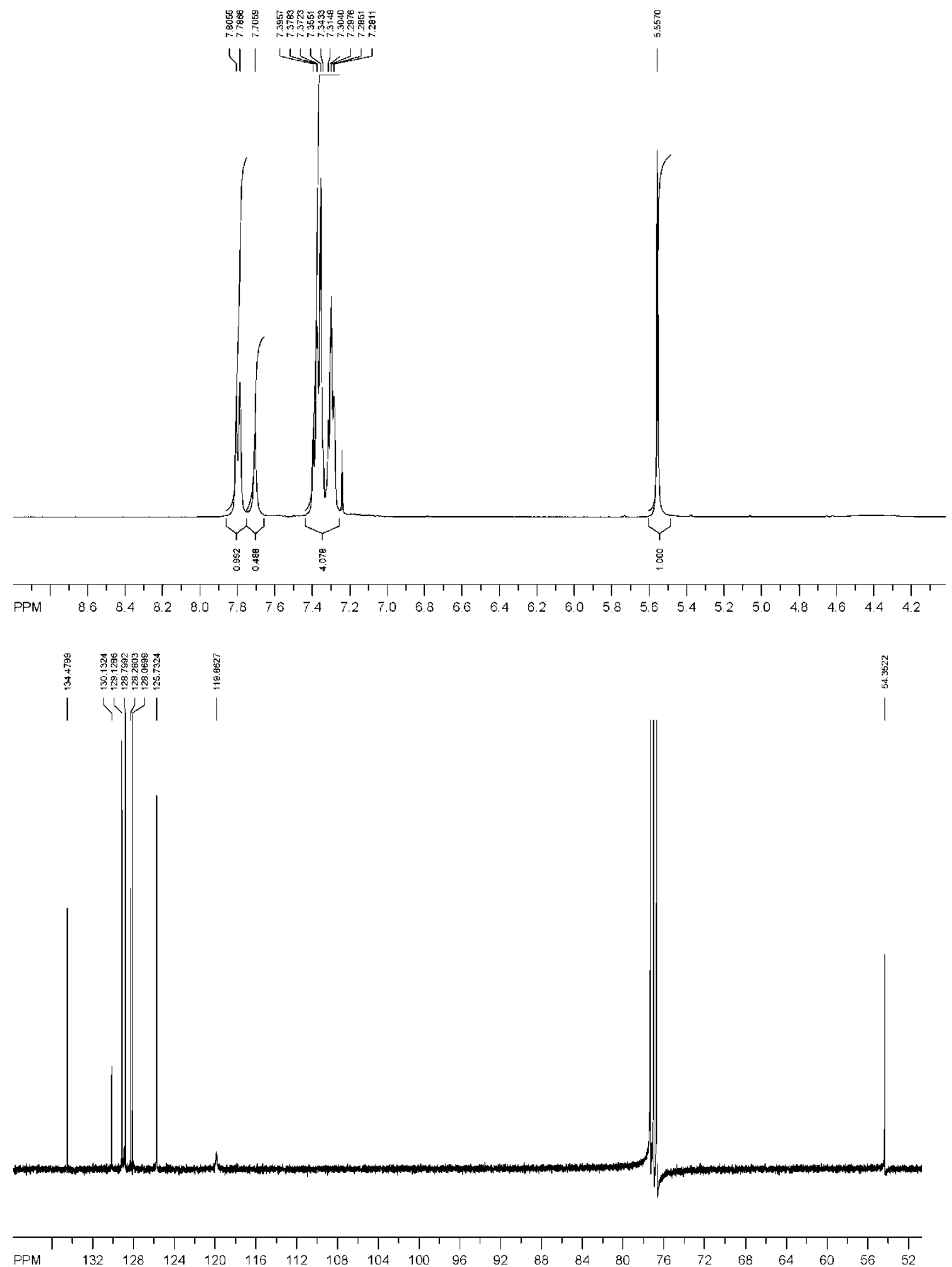
1-(Benzyl)-4-(4-methylphenyl)-1H-1,2,3-triazole (3b):
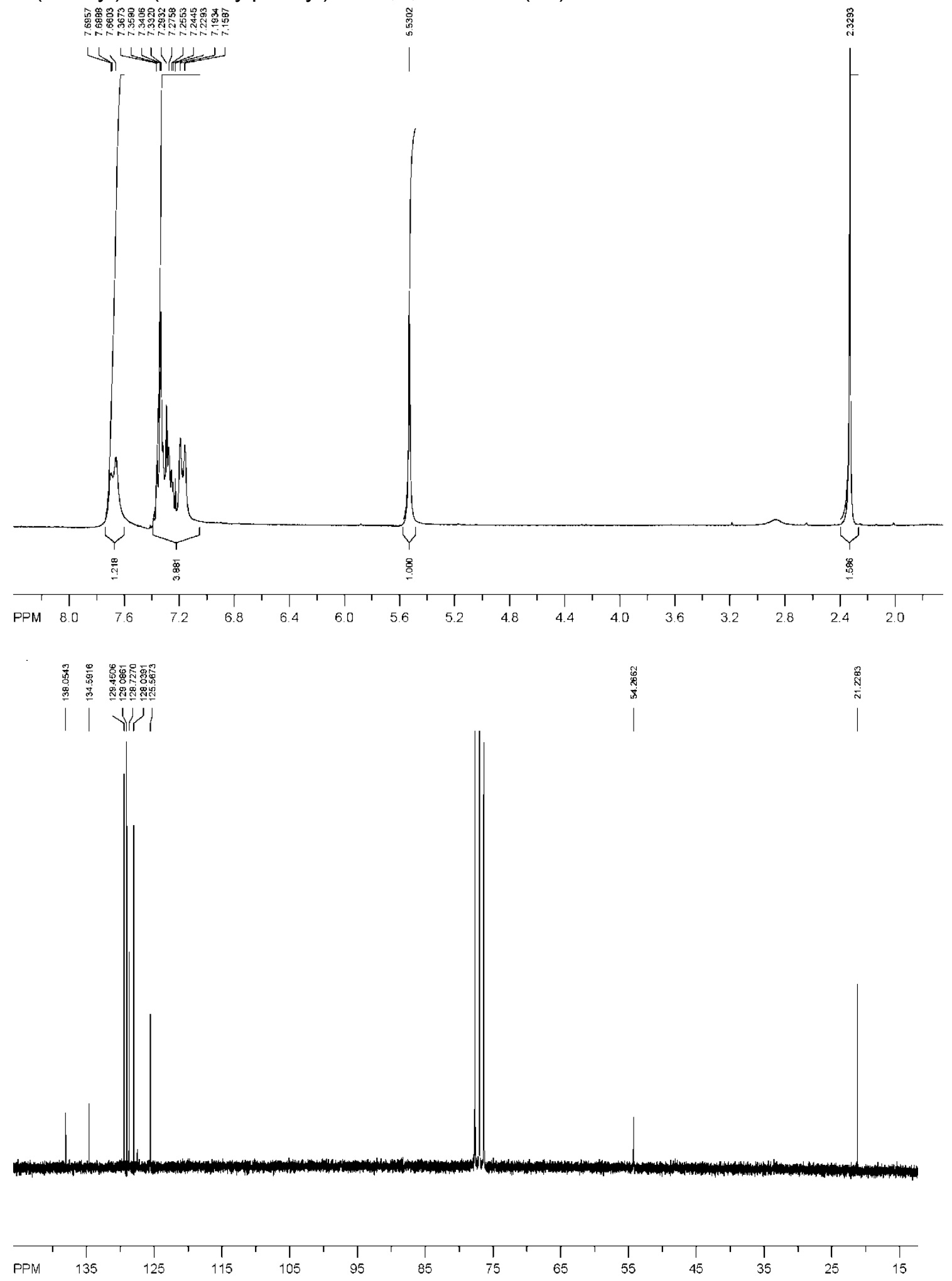
1-(Benzyl)-4-(4-methoxyphenyl)-1H-1,2,3-triazole (3c):

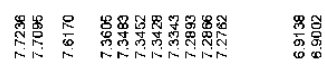

$4 \mid$ ity
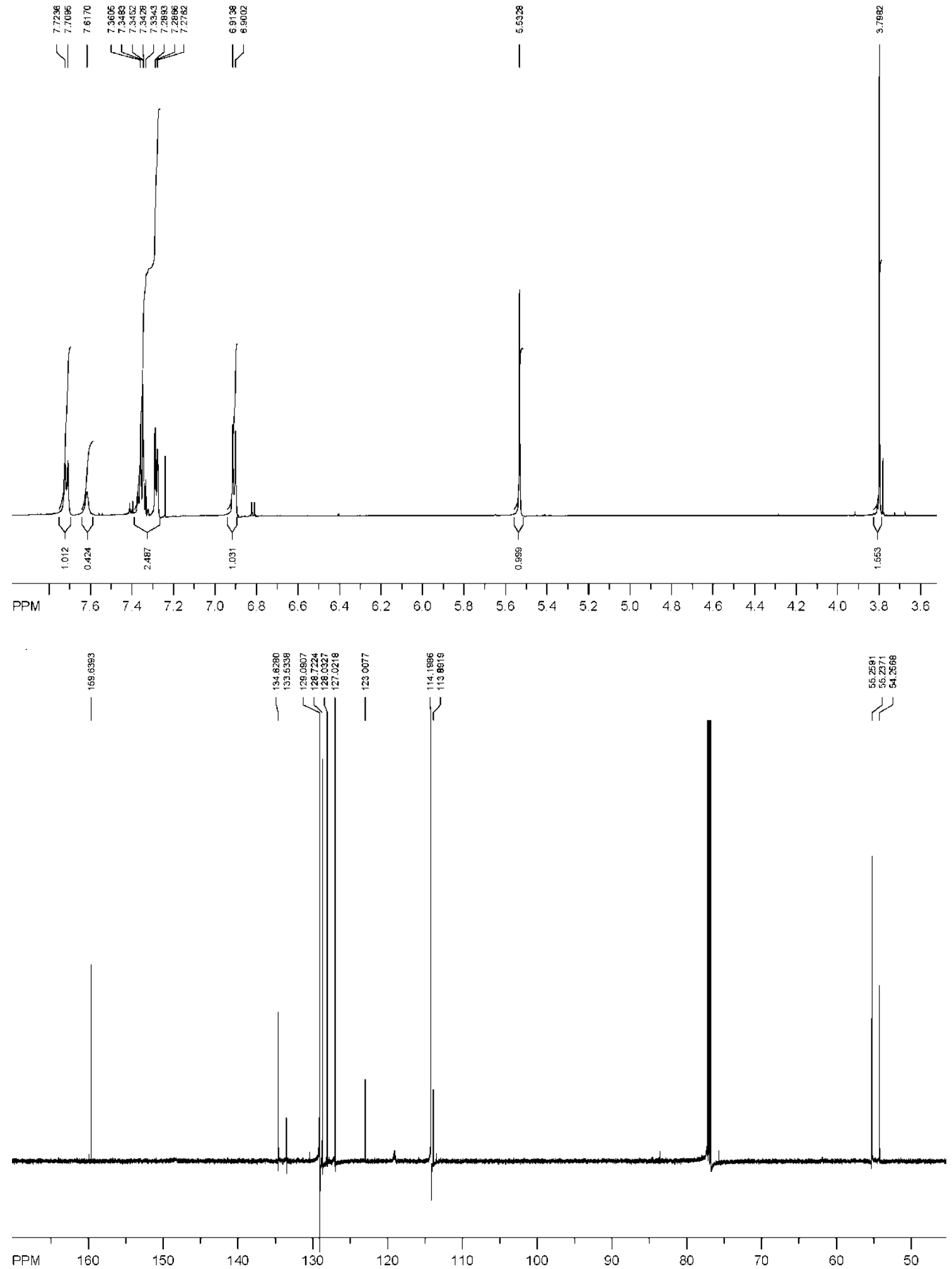
1-Benzyl-4-octyl-1H-1,2,3-triazole (3d):
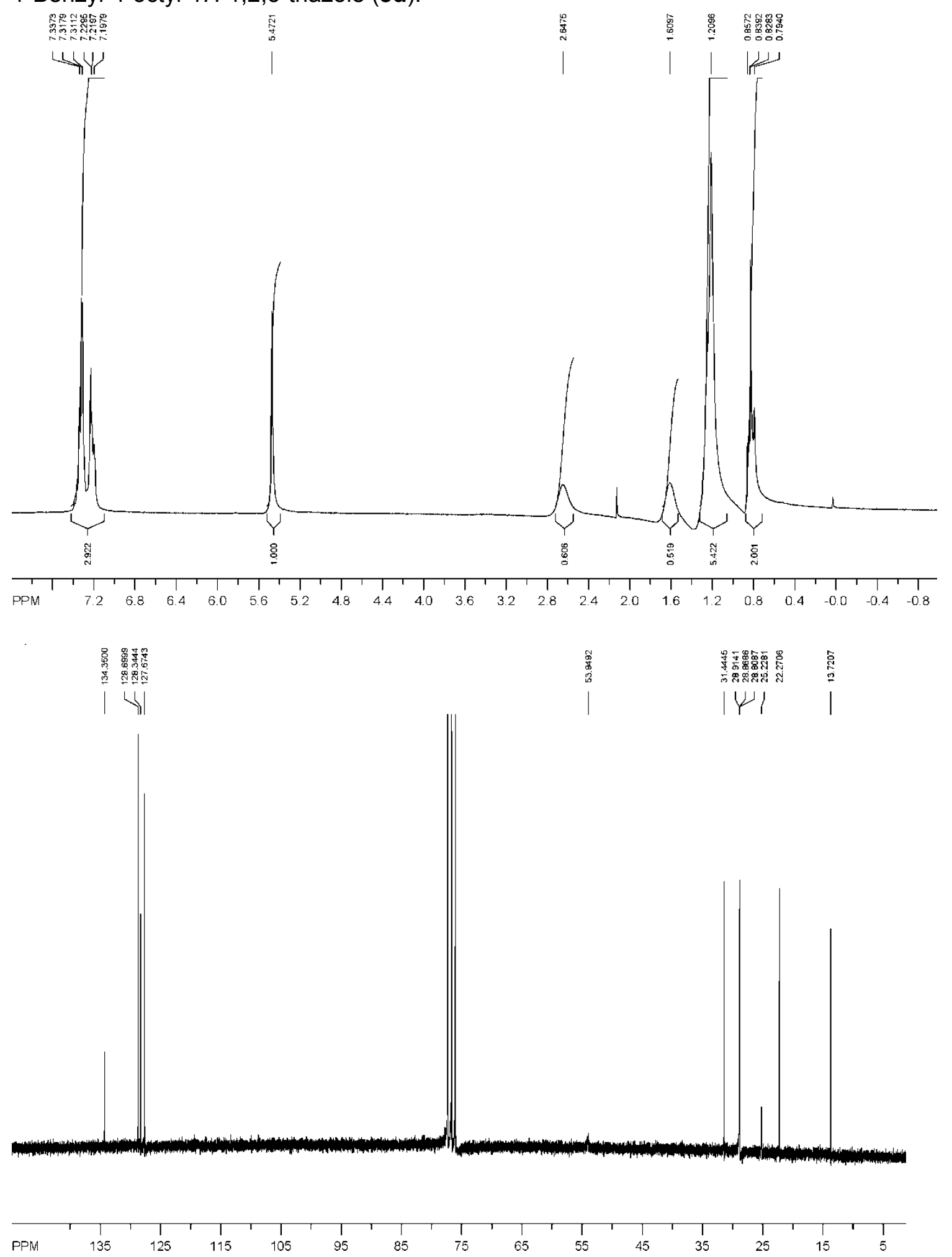
1-Benzyl-4-decyl-1H-1,2,3-triazole (3e):
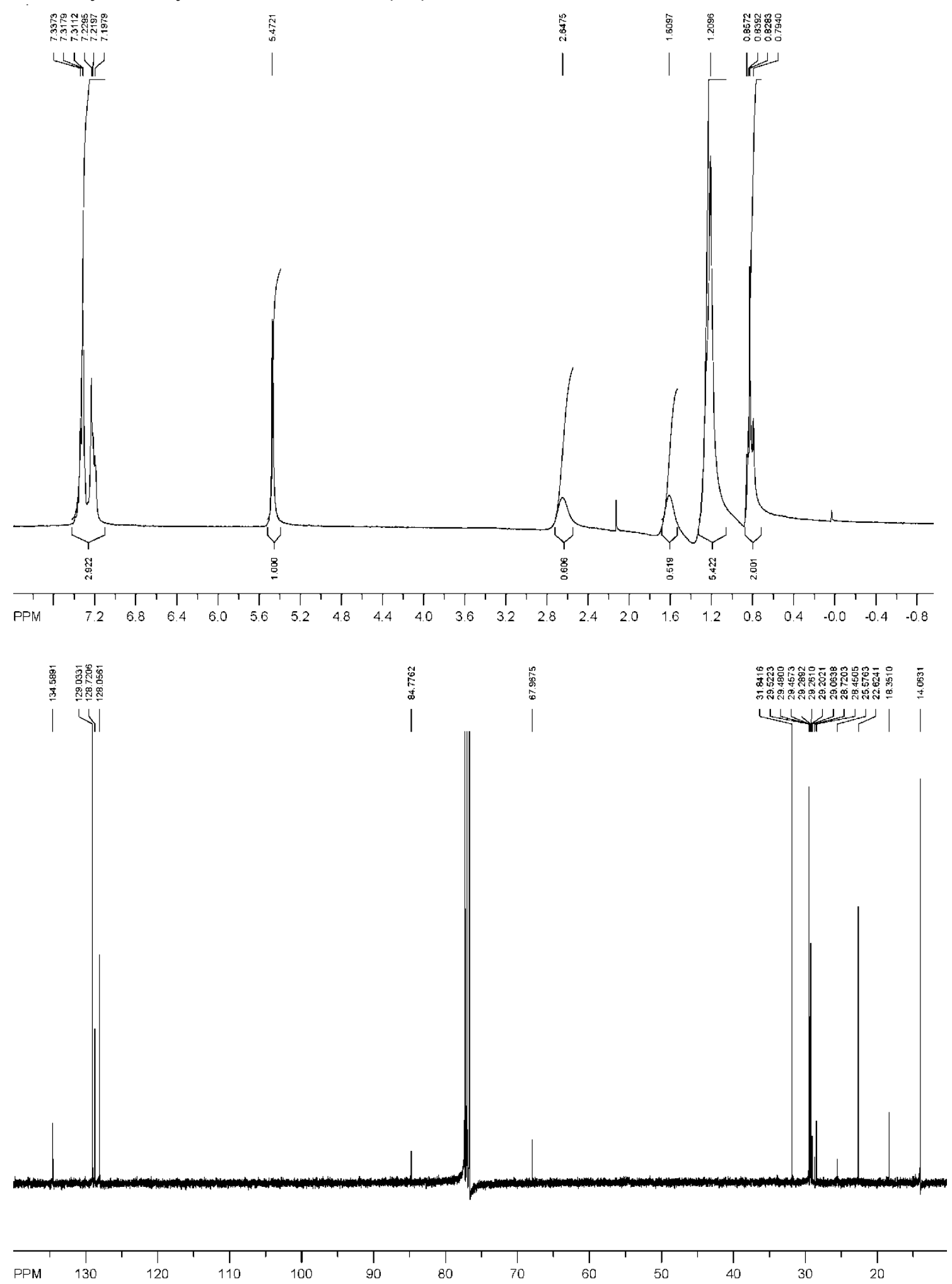
2-(1-benzyl-1H-1,2,3-triazole-4-yl)propan-2-ol (3f):
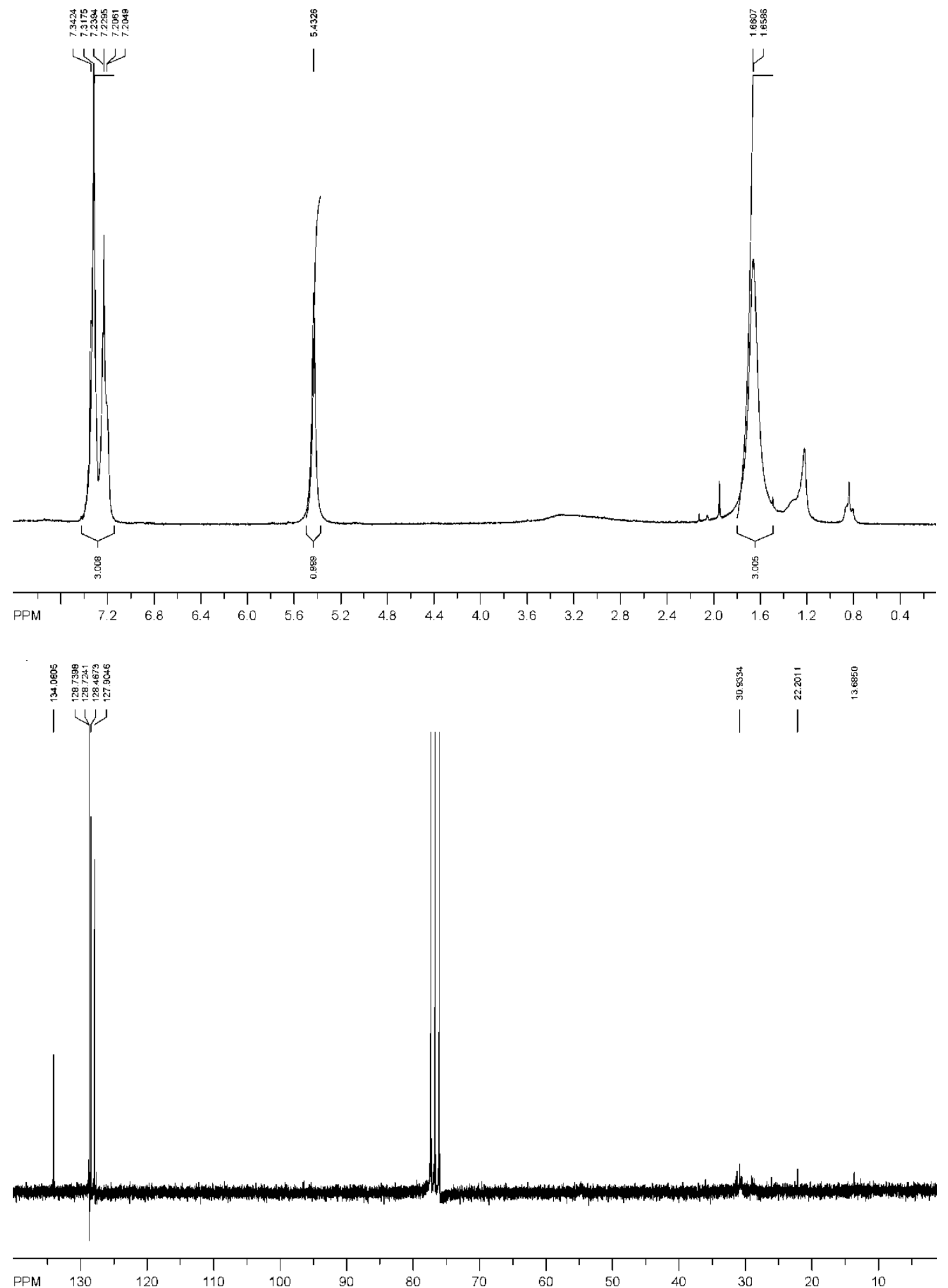
2-(1-benzyl-1H-1,2,3-triazol-4-yl)but-3-en-2-ol (3g):
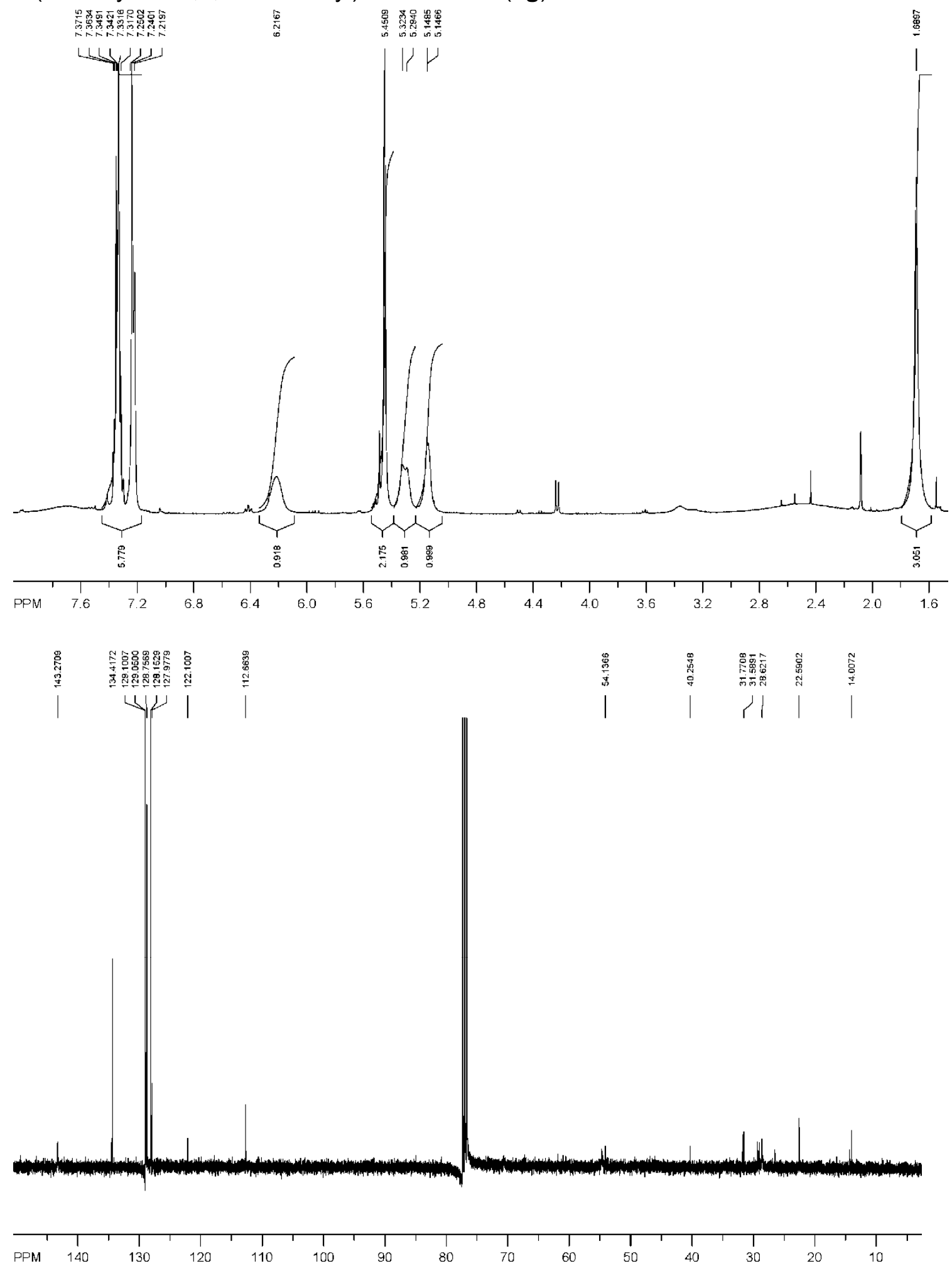
2-(1-benzyl-1H-1,2,3-triazol-4-yl)-6-methylhept-5-en-2-ol (3h):
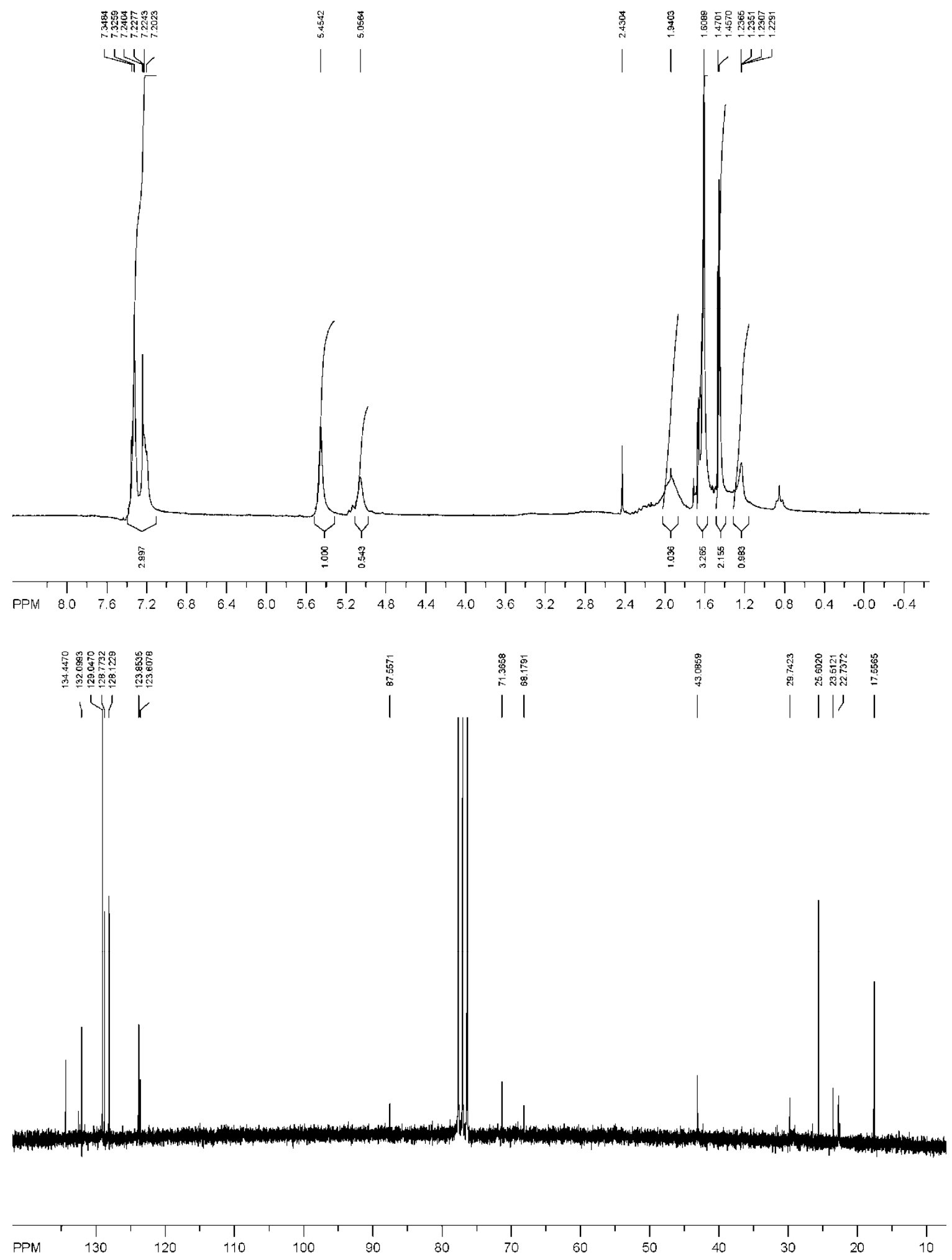
Methyl 1-benzyl-1H-1,2,3-triazole-4-carboxylate (3i):
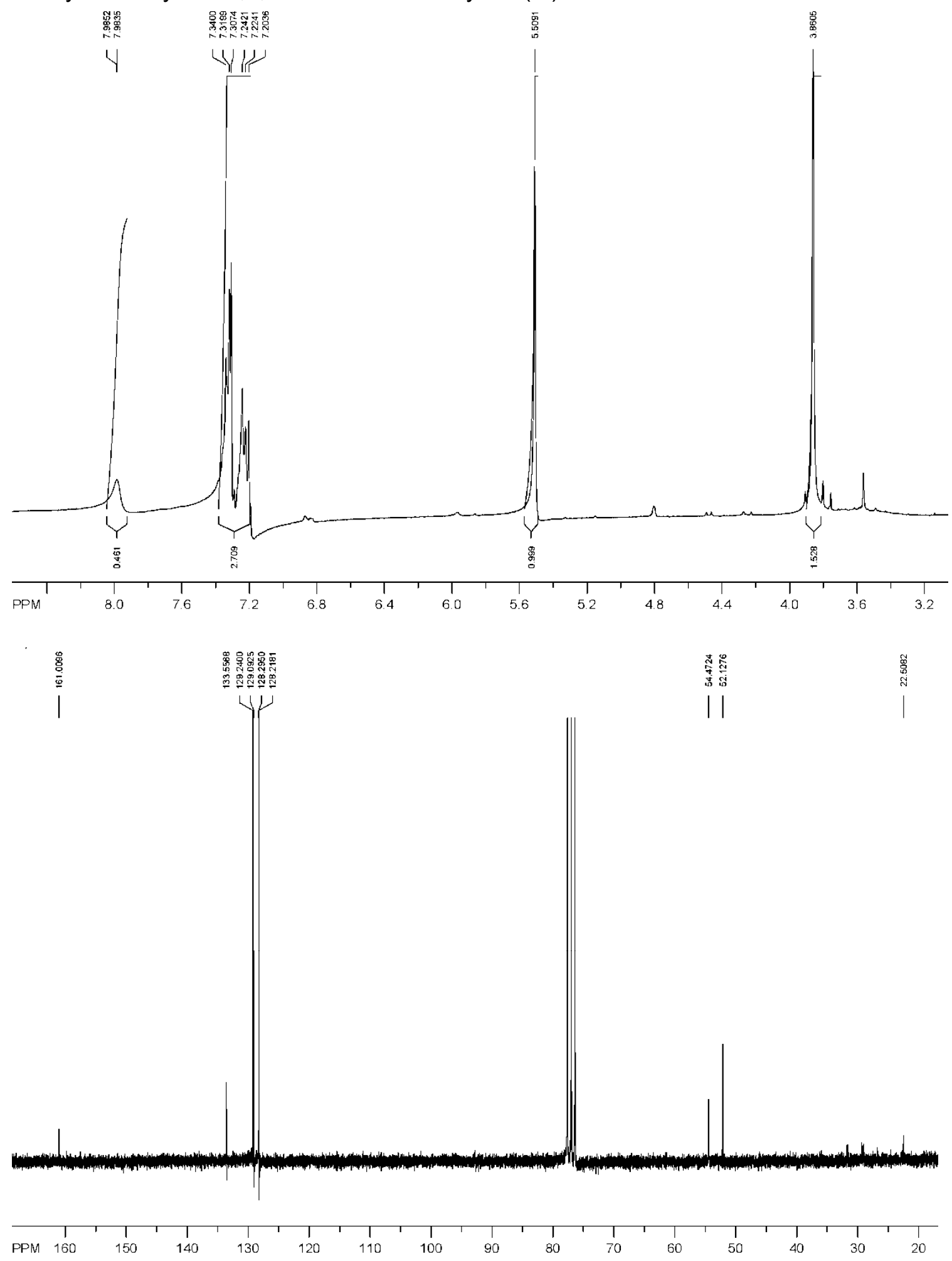
Dimethyl 1-benzyl-1H-1,2,3-triazole-4,5-dicarboxylate (3j):

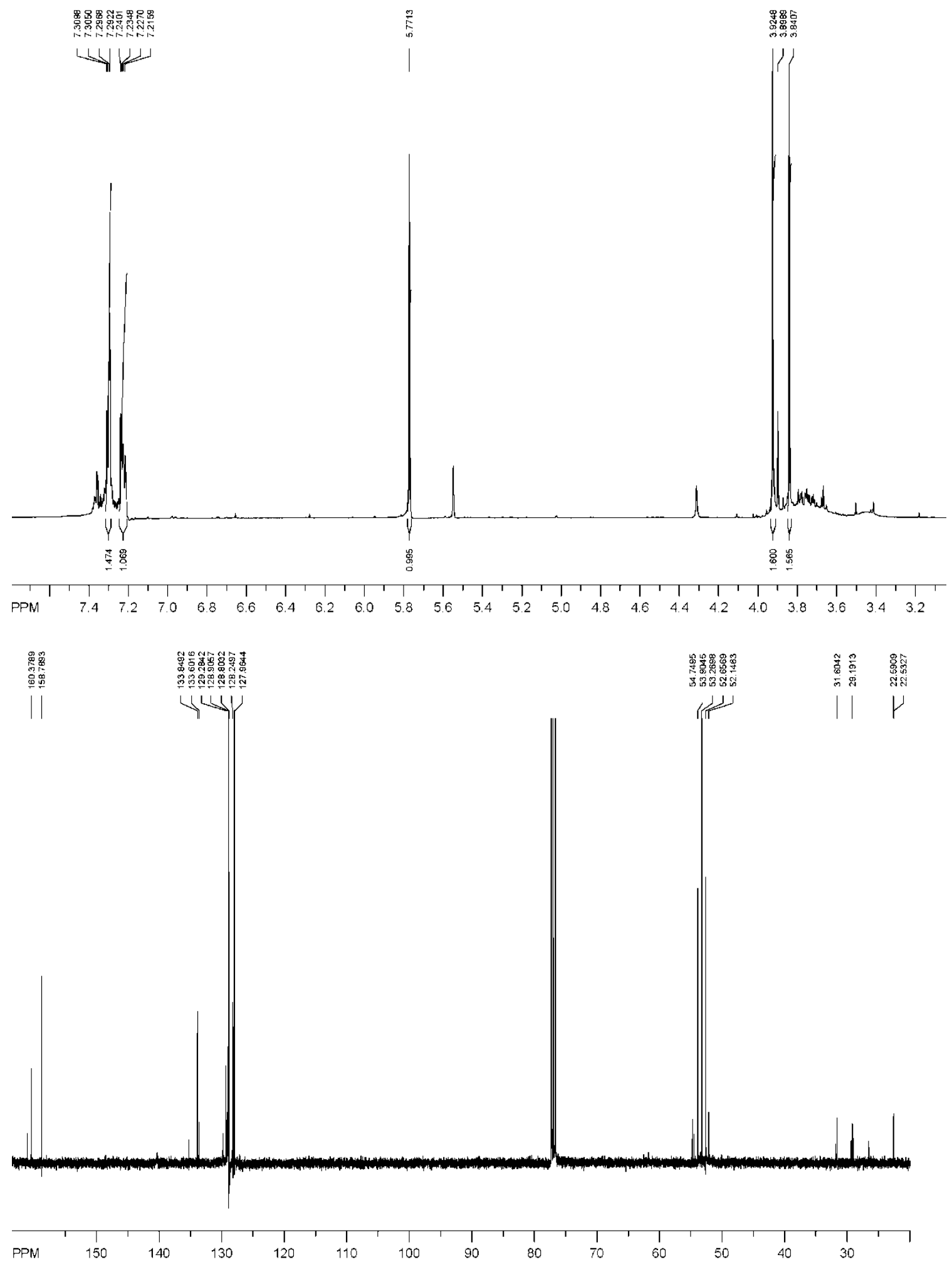


Methyl 1-benzyl-1H-1,2,3-triazole-4-carboxylate (3i; fromreaction of $\mathbf{2 k}$ ):
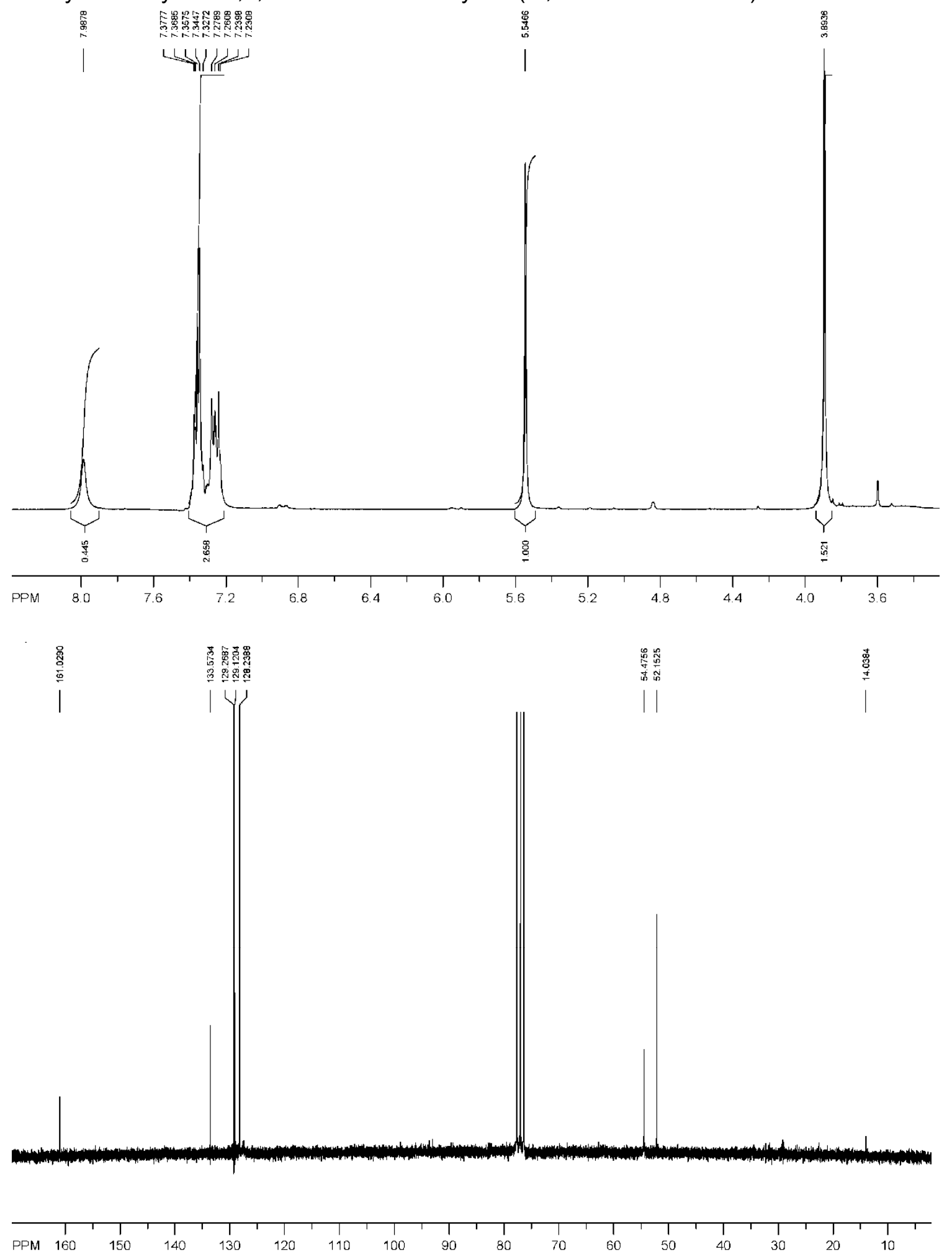
1-Decyl-4-phenyl-1H-1,2,3-triazole (3m):

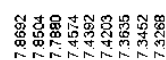

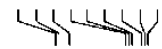

尌喜嘉

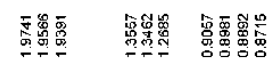

陪

if

$d$

W

듐담 管
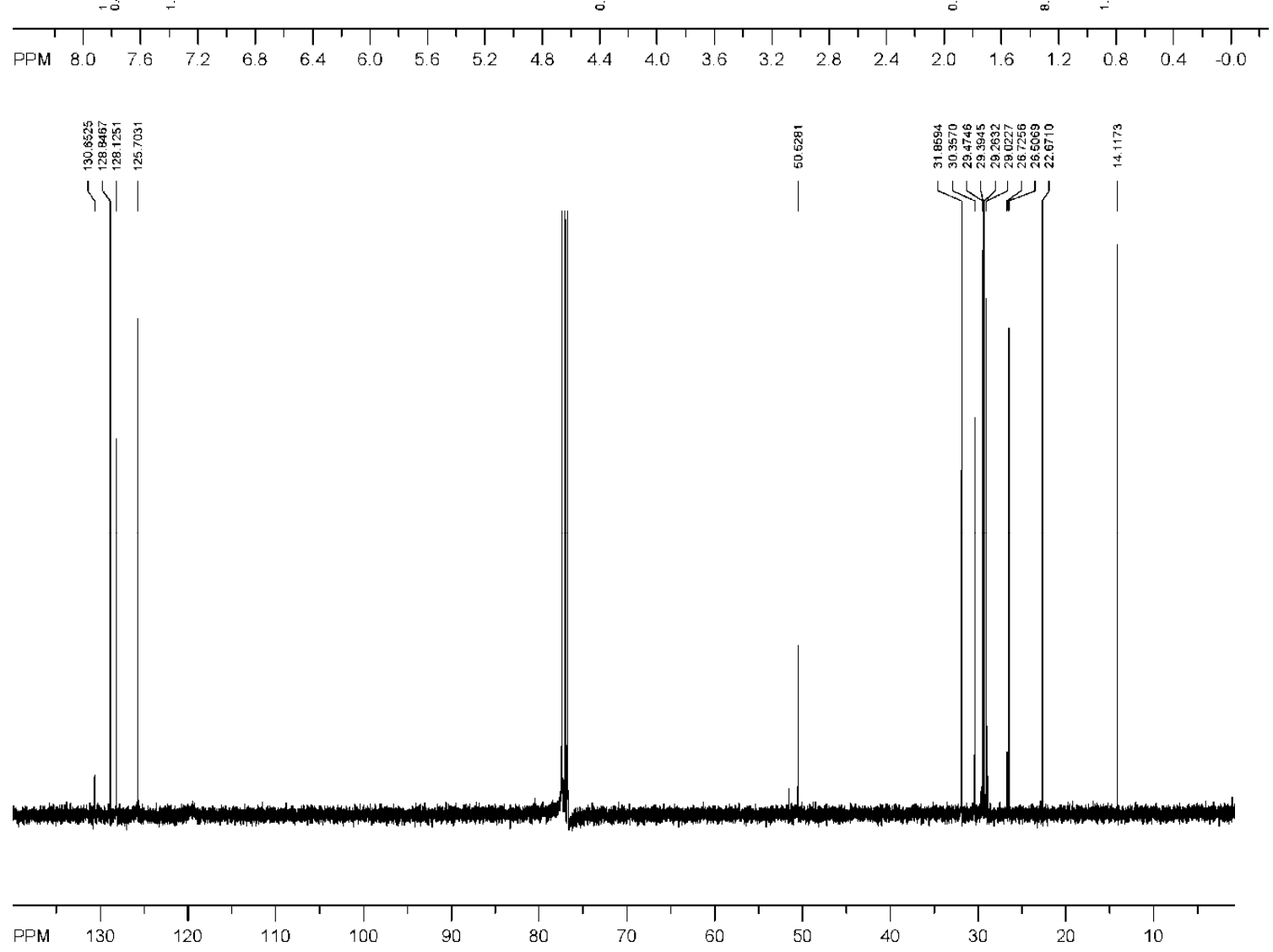